(C) 2001 International Press

Adv. Theor. Math. Phys. 5 (2001) 483-498

\title{
On the Relationship between the
}

\author{
Rozansky-Witten and the
}

\section{3-Dimensional Seiberg-Witten}

\section{Invariants}

\author{
Matthias Blau and George Thompson \\ The Abdus Salam ICTP, \\ P.O. Box 586, \\ 34100 Trieste, \\ Italy \\ mblau@ictp.trieste.it, thompson@ictp.trieste.it
}

\begin{abstract}
The Seiberg-Witten analysis of the low-energy effective action of $d=4 N=2 \mathrm{SYM}$ theories reveals the relation between the Donaldson and Seiberg-Witten (SW) monopole invariants. Here we apply analogous reasoning to $d=3 N=4$ theories and propose a general relationship between Rozansky-Witten (RW) and 3-dimensional Abelian monopole invariants. In particular, we deduce the equality of the $S U(2)$ Casson invariant and the 3-dimensional SW invariant (this includes a special case of the Meng-Taubes theorem relating the SW invariant to Milnor torsion). Since there are only a finite number of basic RW invariants of a given degree, many different topological field theories can be used to represent essentially the same topological invariant. This leads us to advocate using higher rank Abelian gauge theories to shed light on the higher (non-Abelian) RW invariants and we write down candidate higher rank SW equations.
\end{abstract}




\section{Introduction}

Quite a few years ago Witten showed how Donaldson theory finds a natural place in the context of quantum field theory [1]. Formal developments soon uncovered the position of other theories in this pantheon (see e.g. [2] for a review).

After Seiberg and Witten solved the $N=2$ supersymmetric Yang-Mills theory in 4 dimensions [3] the full force of quantum field theory techniques was brought to bear on various mathematical theories. Perhaps the most celebrated result is the (still to be fully established) equivalence of the 4dimensional Seiberg-Witten monopole invariants [4] and the Donaldson invariants.

The purpose of this paper is to apply analogous reasoning to 3-dimensional supersymmetric gauge theories and their associated invariants. The relationship between the 4- and the 3-dimensional theories has been analysed in great detail in [5]. We stay firmly in 3 dimensions.

One of our motivations was to understand a theorem of Meng and Taubes from a physics point of view. Their theorem equates the Seiberg-Witten invariants of a 3-manifold $M$ with its Milnor torsion, providing $b_{1}(M) \geq 1$ [6]. On the other hand it is known that the Casson invariant with the same homological constraint on $M$ is given in terms of the Alexander polynomial of $M$ [7]. The relationship between Milnor torsion and the Alexander polynomial [8] means that the Seiberg-Witten invariant equals the Casson invariant (for $b_{1} \geq 1$ ). Why should this be so? Amongst other things we answer this question.

We focus on $N=4$ supersymmetric Yang-Mills theories with gauge group $G$ and combine the following well known facts about these theories:

1. The Coulomb branch of any $N=4$ SYM theory in 3 dimensions is a (possibly singular) hyper-Kähler manifold [9].

2. Topologically twisting the low-energy description of this theory one obtains the perturbative Rozansky-Witten [10] (or generalized Casson) invariants.

3. Topologically twisting the microscopic theory instead, one obtains a topological field theory with two topological charges, $N_{T}=2$, modelling the de Rham complex and formally calculating an Euler characteristic of some gauge theory moduli space $[11,12,13,14]$. 
The two topological field theories, the one calculating the Euler characteristic and the other yielding perturbative invariants, arise on twists of the same physical theory albeit at different energy scales. Since the topological theory should not care what scale one is working at one immediately arrives at the equality of the two types of topological field theories. This is the same reasoning that one uses to establish that the Seiberg-Witten invariants on a 4-manifold are equivalent to the Donaldson polynomial invariants.

The paradigm then is that the topological invariants that arise from twisting the original gauge theory match the topological invariants that arise on twisting the low energy effective theory.

In 3 dimensions, this reasoning leads to non-trivial results even in the case that the gauge group of the microscopic theory is Abelian. Indeed, as we will see below, applying this reasoning to the $N=4 U(1)$ theory with $N_{f}=1$ hypermultiplet yields the equivalence of the 3-dimensional version of the Seiberg-Witten monopole invariants and the Casson (actually CassonLescop-Walker) invariant, not just for $b_{1} \geq 1$ (this is part of the content of the Meng-Taubes theorem) but also for the (mathematically much more subtle) case $b_{1}=0$.

But this is just the first in a whole hierarchy of observations one can deduce in this way. The crucial additional input is the fact that, denoting the hyper-Kähler manifold apapearing as the target space of the RozanzkyWitten sigma model by $X, \operatorname{dim}_{\mathbb{R}} X=4 n$,

4. there are only a finite number of independent perturbative ${ }^{1}$ invariants for each $n$. For example the number of independent perturbative invariants for $n=1,2,3,4$, are $1,1,1,2$.

Combining these facts one realises that many (possibly infinitely many) different gauge theoretic moduli spaces have Euler characteristics which (as functions of the 3-manifold) are linearly dependent.

The main mystery of the perturbative invariants is their relationship to data of the 3-manifold. The Rozansky-Witten viewpoint provides a resolution to this dilemma as it relates generalized Casson invariants to the perturbative invariants. The Casson invariants are tied to $\pi_{1}(M)$ and so the finite

\footnotetext{
${ }^{1}$ In the following we refer to the Rozansky-Witten invariants as perturbative or finite type invariants. However, that the RW invariants are of finite type has not been rigorously established even though there is a lot of evidence in favour of this. All we really need is that there are a finite number of RW invariants at any order in perturbation theory and this is obviously true.
} 
type invariants must also know about the fundamental group. However, our point of view is that the Rozansky-Witten approach offers a plethora of different moduli space interpretations of the finite type invariants. Ultimately, it may well be that the most interesting information is the relationship to the fundamental group of $M$. Nevertheless, the availability of the many different representations of the invariants offers computational power. One general conclusion is that it is worthwhile to try to simplify the situation as much as possible. The Seiberg-Witten invariants on a 4-manifold are analytically more tractable than the Donaldson invariants. Likewise, rather than studying the $S U(n)$ Casson invariants directly, it may well be profitable to look at the associated Abelian Seiberg-Witten equations. We take a preliminary look at such equations in section 3.2.

There remains a mystery, however. Why do all the gauge theoretic moduli spaces lead to perturbative, presumably finite type, invariants?

\section{The Casson, RW and SW Invariants}

In this section we make use of the identification of the various topological field theories to relate their associated topological invariants. We concentrate on those theories which have as their Coulomb branch moduli space some 4-dimensional hyper-Kähler space. Given a hyper-Kähler manifold $X$ of real dimension 4 the associated Rozansky-Witten invariant for a 3-manifold $M$ is, in the notation of [15],

$$
Z_{X}^{R W}[M]=\mathbf{e}(X) \lambda(M)
$$

where $\mathbf{e}(X)$ is the integral of the Euler class ${ }^{2}$ of $X$ and $\lambda(M)$ is the suitably normalised Casson-Lescop-Walker invariant [16], [7] (which extends the Casson invariant, originally defined for integral homology spheres, to all 3manifolds).

By the discussion in the Introduction we learn that any $N=4$ theory with a 4-dimensional Coulomb branch yields (upon twisting) a topological invariant proportional to the Casson invariant. This includes gauge theories with group $U(1)$ and any number of charged hypermultiplets or the gauge group $S U(2)$ and any number of fundamental hypermultiplets. In the list of such theories one possibly also has examples with compact Coulomb branches, obtained via toroidal dimensional reduction [17]. As the only perturbative invariant available is the Casson invariant, the Rozansky-Witten

\footnotetext{
${ }^{2}$ Note that for $X$ non-compact this is not necessarily the Euler characteristic.
} 
invariants associated to all of these theories are proportional to the Casson invariant.

In turn this means that the wildly different moduli spaces associated with the gauge theories all have Euler characteristic proportional to the Casson invariant, the proportionality factor being $\mathrm{e}(X)$. For example the $U(1)$ gauge theory with one charged hypermultiplet, when twisted, yields the topological field theory corresponding to the 3-dimensional Seiberg-Witten equations. The pure $S U(2)$ theory yields the Casson invariant [10], while the $S U(2)$ theory with one hypermultiplet is the topological field theory for the $S U(2)$ Seiberg-Witten monopole moduli space. We take a look at all of these spaces next.

\subsection{The Casson and the RW Invariants}

The starting physical theory is the pure $N=4$ supersymmetric $S U(2)$ gauge theory. It has been argued that this theory, when twisted, calculates the Casson invariant (this essentially goes back to Taubes' gauge theoretic interpretation of the Casson invariant [18] which was given a topological field theory interpretation in [19] subsequently elaborated upon in [11]). On the other hand the low energy effective theory has as its moduli space the AtiyahHitchin manifold $X_{A H}$ [9], which is the $S U(2)$ 2-monopole moduli space. It is known that

$$
\mathbf{e}\left(X_{A H}\right)=1,
$$

and so the Rozansky-Witten (1) invariant in this case really is equal to the Casson invariant, as it should be!

\subsection{The Seiberg-Witten and the RW Invariants}

Consider a $N=4, U(1)$, supersymmetric gauge theory with $N_{f}=1$ hypermultiplets. Seiberg and Witten have shown that the moduli space is the Taub-Nut hyper-Kähler manifold $X_{T N}$ [9]. If we now twist this theory we obtain a supersymmetric sigma model with target space $X_{T N}$. The integral of the Euler class of the Taub-Nut is

$$
\mathrm{e}\left(X_{T N}\right)=1 .
$$

The Rozansky-Witten invariant is therefore once more equal to the Casson invariant. 
On the other hand, as we will now see, the topological invariant associated with the microscopic theory counts solutions to the 3-dimensional Abelian Seiberg-Witten monopole equations.

The gauge theory setting is that of $N=4$ supersymmetric Yang-Mills theory with gauge group $U(1)$ equipped with a charged hypermultiplet. The $N=4$ theory is most usefully regarded as the dimensional reduction of the six-dimensional $N=1$ theory to 3 dimensions. This exhibits the Rsymmetry group $S U(2)_{R} \times S U(2)_{N}$ of the 3-dimensional theory, $S U(2)_{R}$ being the R-symmetry group of the six-dimensional theory and $S U(2)_{N}$ the rotation group in the 'internal' 3 dimensions.

The fields in the charged hypermultiplet transform as

$$
\text { Bosons : }(2,1,1)^{ \pm}, \text {Fermions : }(1,2,2)^{ \pm} \text {, }
$$

under $S U(2)_{R} \times S U(2)_{N} \times S U(2)_{E} \times U(1)$ where $S U(2)_{E}$ is the space-time Lorentz group (for a more careful discussion of R-symmetry groups in the Euclidean versus Lorentzian theories and their twists see [20] - we will not have to worry about these issues here).

Now what theory do we have if we twist the model directly without passing to the low energy theory? Twisting in this case means that we consider the diagonal, $S U(2)_{E^{\prime}}$ of $S U(2)_{R} \times S U(2)_{E}$ to be the new Lorentz group. From (4) we see that after twisting the field content transforms as

$$
\text { Bosons : }(1,2)^{ \pm}, \quad \text { Fermions : }(2,2)^{ \pm} \text {, }
$$

under $S U(2)_{N} \times S U(2)_{E^{\prime}} \times U(1)$. This, together with the twisted vector multiplet, is precisely the field content of the topological theory corresponding to the 3-dimensional Seiberg-Witten equations and twisting the supersymmetric action leads us directly to the action for the topological theory $[21,23,22]$.

Denoting the gauge field by $A$, its field strength by $F$, and the (commuting) spinor field arising from the twisted hypermultiplet by $M$, the 3dimensional SW equations are

$$
\begin{aligned}
F_{\mu \nu} & =-\frac{i}{2} \bar{M} \sigma_{\mu \nu} M \\
\not D(A) M & =0 .
\end{aligned}
$$

(We will occasionally write the first equation more compactly as $F_{A}=$ $* \bar{M} \gamma M$.)

In this way we have established that the physics of $N=4$ gauge theories in 3 dimensions predicts

$$
S W(M)=\lambda(M)
$$


The reader should note that we are summing over all $\mathrm{U}(1)$ bundles in the path integral. On the Seiberg-Witten side, as explained in [4] this is

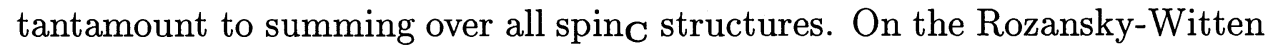
side it is this sum which dualizes the photons turning them to compact scalars which combine, in turn, with the other scalars to form the hyperKähler manifold.

\subsection{Evidence in Favour of SW = Casson}

There is abundant evidence in the mathematics literature that supports this claim. Meng and Taubes have shown that for $b_{1}(M) \geq 1$

$$
\underline{\mathrm{S} W}\left(M, t_{i}\right)=\tau\left(M, t_{i}\right)
$$

where $\tau\left(M, t_{i}\right)$ is the Milnor torsion of $M$ while the SW series $\underline{\mathrm{SW}}\left(M, t_{i}\right)$ of $M$ is defined e.g. in $[6,5]$. As a special case, the only one we will actually need, we have

$$
\mathrm{S} W(M)=\tau(M, 1),
$$

where $\mathrm{S} W(M)=\underline{\mathrm{S} W}\left(M, t_{i}=1\right)$ and $\tau(M, 1)=\tau\left(M, t_{i}=1\right)$. For $b_{1}(M)>1$ one has

$$
\tau\left(M, t_{i}\right)=\Delta_{M}\left(t_{i}\right),
$$

where $\Delta_{M}\left(t_{i}\right)$ is the Alexander polynomial of $M$, symmetrized in $t$ and $t^{-1}$. Lescop [7] has shown for $b_{1}(M)>1$ that $\lambda(M)=\Delta_{M}\left(t_{i}=1\right)$. Consequently, for $M$ such that $b_{1}(M)>1$

$$
\mathrm{S} W(M)=\lambda(M)
$$

For $b_{1}(M)=1$ the relationship between the Milnor Torsion and the Alexander polynomial is [8]

$$
\tau(M, t)=\frac{t \Delta_{M}(t)}{(1-t)^{2}}
$$

The right hand side expanded about $t=1$,

$$
\frac{t}{(1-t)^{2}} \Delta_{M}(1)+\frac{t}{2} \Delta_{M}^{(2)}(1)+\ldots
$$

is singular as $t \rightarrow 1$ and so must be suitably interpreted. Note that

$$
\frac{1}{(1-t)^{2}}=\frac{d}{d t} \frac{1}{(1-t)}=\frac{d}{d t} \sum_{n=0}^{\infty} t^{n}=\sum_{n=1}^{\infty} n t^{n-1}
$$


which, as $t \rightarrow 1$, goes over to

$$
\lim _{s \rightarrow-1} \sum_{n} n^{-s}=\zeta(-1)=-\frac{1}{12} .
$$

The regularised form of the limit is then

$$
\tau(M, 1)=-\frac{1}{12} \Delta_{M}(1)+\frac{1}{2} \Delta_{M}^{(2)}(1)
$$

which agrees, once more, with the result found by Lescop for the Casson invariant.

The most important case is when $b_{1}(M)=0$, i.e. when $M$ is a rational homology 3 -sphere. It has been shown that for integral homology spheres the SW invariant equals the Casson invariant [24]. This is conjectured to be the case also for rational homology spheres and there is considerable evidence for this [27].

Actually there is a subtlety here. The Seiberg-Witten equations are defined with some perturbation. For $M$ a rational homology sphere one deforms the equations to

$$
F_{A}=* \bar{M} \gamma M+d \nu
$$

where $\nu$ is a 1 -form on $M$. With this choice of perturbation there is a choice of metric such that the reducible solution set $(A, M)=(\nu, 0)$ is isolated from the irreducible solutions (which themselves form a finite set). The problem is that this split is not so clean. As one varies the metric and the perturbation, some of the irreducible points may collide with the reducible solution, or some irreducible points may bubble off from the reducible solution. This means that the count of the signed sum of the irreducible points is not an invariant. On the other hand the 'total' path integral is formally metric and perturbation invariant. The problem seems to have arisen because we have 'excised' the reducible.

So the path integral may well be defined to count the reducible point (in some fashion, depending on the metric and on the perturbation) together with a signed sum of the irreducible points. The usual BRST argument suggests that one can change the metric and perturbation at will, providing the solution set remains finite. In this case extra solutions to the Dirac equation may appear at the reducible as one varies the parameters and a correction term is required to compensate the dissapearance of solutions away from the reducible. 
There is a choice for the contribution of the reducible connection in terms of $\eta$-invariants (see e.g. [25, 24, 26]),

$$
\frac{1}{2} \eta\left(\not D_{\nu}\right)+\frac{1}{8} \eta(* d-d *)
$$

which is known to ensure that the total contribution yields the Casson invariant for integral homology 3 -spheres. The same contribution is conjectured to be correct for rational homology 3-spheres as well and there is some evidence to support this [27]. Note incidentally that for rational homology spheres admitting a metric of positive scalar curvature (e.g. Lens spaces) the above correction term is the only contribution to the SW invariants as there are no non-trivial solutions to the SW monopole equations in this case.

It is interesting to note that the Rozansky-Witten invariant is affected by an analogous 'correction' term. In this case the ambiguity amounts to a choice of 2 -framing of $M$. That choice must be fixed for the invariant to coincide with $\lambda(M)$.

\subsection{The SU(2) Theory with Hypermultiplets}

Seiberg and Witten also studied the $S U(2)$ supersymmetric theory with $N_{f}$ hypermultiplets in the fundamental representation [9]. The Coulomb branch moduli space depends on $N_{f}$. They give quite a complete description of the spaces involved:

1. For $N_{f}=1$ the space is $\tilde{X}_{A H}$, the double cover of $X_{A H}$.

2. For $N_{f}=2$ the space is topologically and metrically $\left(\mathbb{R}^{3} \times S^{1}\right) / \mathbb{Z}_{2}$.

3. For $N_{f}>2$ they are ALE spaces with a $D_{N_{f}}$ singularity, $\mathbb{C}^{2} / \Gamma_{N_{f}-2}$.

For $N_{f} \geq 2$ one should perhaps resolve the singularities and this can be achieved by adding bare mass terms to the hypermultiplets. The corresponding Rozansky-Witten invariants are

1. $N_{f}=1: 2 \lambda(M)$.

2. $N_{f}=2: 0$.

3. $N_{f}>2:\left(2 N_{f}-1-\frac{1}{4\left(N_{f}-2\right)}\right) \lambda(M)$. 
We now need to see what moduli space one gets on the gauge theory side. The reader will not be too surprised to learn that the moduli space of the topological gauge theory is that of non-Abelian monopoles. Non-Abelian monopole equations have been studied in 4 dimensions [29] and one may just as well consider them in 3 dimensions. Let $\mathcal{S}$ denote the spin bundle and $E_{i}$ be complex vector bundles associated to a principal $G$-bundle via the representations $R_{i}$. Let $M_{i}$ be sections of $\mathcal{S} \otimes E_{i}$. The monopole equations are

$$
F_{A}^{a}=* \sum_{i=1}^{N_{f}} \bar{M}_{i} \gamma T_{i}^{a} M_{i}, \quad \not D_{A} M_{i}=0
$$

where $T_{i}^{a}$ are the generators of the Lie algebra of $G$ in the representation $R_{i} . \quad G=S U(2), \operatorname{rank}(E)=2$ and $N_{f}=1$ is the case most studied in 4 dimensions. The situation with bare mass terms for the hypermultiplet included has also been considered in that context.

The claim now is that the Euler characteristic of the non-Abelian $S U(2)$ monopole equations (20) (suitably perturbed) equals the multiple of the Casson invariant listed above.

\section{Higher Rank Seiberg-Witten Invariants}

We have, so far, only concentrated on theories which are proportional to the usual Casson invariant or, equivalently, to those which have a 4-dimensional Coulomb branch moduli space. Considering a group of larger rank or adding matter content in other representations (for example the adjoint representation) leads to a higher dimensional hyper-Kähler space. From the RozanskyWitten side this means that the invariant being probed is a higher order RW invariant.

In order to see the higher order RW invariants certain integrals of products of the Riemann curvature tensor on the hyper-Kähler space must be non-zero. Roughly one has a dependence of the type (see equation (10.17) in [15])

$$
\lambda_{X}^{k}(M)=B_{i}(X) \lambda_{i}^{k}(M)+\ldots
$$

where $\lambda_{i}^{k}(M)$ is the 3-manifold dependence of the $i$-th RW invariant of order $k, B_{i}(X)$ is the dependence on the hyper-Kähler manifold (it also depends on $\left.b_{1}(M)\right)$ and the ellipses denote dependence on products of lower order 
RW invariants. For $b_{1}(M)>1$ one only needs $\mathbf{e}(X) \neq 0$

$$
\lambda_{X}^{k}(M)=\mathbf{e}(X)(\lambda(M))^{k}
$$

and the ellipses in (21) are zero. For $b_{1}(M)=1$ one finds that the invariant is $[15]$

$$
\lambda_{X}^{k}(M)=\int_{X} \hat{\mathbf{A}}(X) \prod_{i=1}^{n} \Delta_{M}\left(e^{x_{i}}\right),
$$

where the $x_{i}$ are the eigenvalues of the curvature 2-form of the holomorphic tangent bundle. Expanding the $\hat{A}$-genus and $\Delta_{M}$ in terms of the $x_{i}$ and keeping only the top-form components, one finds an expression of the form (21). The dependence on the 3-manifold is through classical invariants and the dependence on the hyper-Kähler manifold is through characteristic classes.

The case of $b_{1}(M)=0$ is quite different. The dependence on the hyperKähler manifold is not just through characteristic classes of the holomorphic tangent bundle [15] but has a rather more subtle dependence on the curvature tensor [28]. Also the dependence on the 3-manifold is not through classical invariants, which is just as well as otherwise there would be nothing new here.

The upshot is that one will have to make judicious choices of the content of the gauge theory to 'see' the higher order invariants. It is believed that the pure $S U(n), N=4$ theories have Coulomb branches which probe some of these invariants.

Incidentally these observations in a sense go both ways. If, for some reason, the 3-manifold invariants in question are known, then one can use the above reasoning to obtain some information on the curvature integrals $B_{i}(X)$ of hyper-Kähler manifolds instead.

For example, 'higher knowledge' from 4-dimensions suggests (at least for large enough $b_{1}(M)$ ) that the Casson invariant should equal the invariant that one obtains from the dimensional reduction of the theory describing the 4-dimensional Seiberg-Witten equations. The reduced theory is that for the 3-dimensional Seiberg-Witten equations. We would deduce, therefore, that $\mathbf{e}\left(X_{T N}\right)=1$. That the reduced invariants come out right has been shown by Mariño and Moore [5]. Similarly the reduction of the Donaldson theory itself must yield the Casson invariant and so we find $\mathbf{e}\left(X_{A H}\right)=1$. Happily both 'predictions' hold and the circle of ideas could have led us to predict that $\mathbf{e}\left(X_{T N}\right)=\mathbf{e}\left(X_{A H}\right)$. 
Perhaps reasoning of this type in other settings, in particular for $b_{1}(M) \geq$ 1 where the RW invariants are classical 3-manifold invariants, could be used to garner information about curvature integrals on other hyper-Kähler manifolds.

\section{1 $b_{1}(M) \geq 1$ and Implications of the Theorem of Meng and} Taubes

We start by exploring the higher order RW invariants for 3-manifolds with $b_{1}(M) \geq 1$. We can rewrite the Rozansky-Witten expression for any $M$ with $b_{1}(M) \geq 1$ in the following compact form

$$
\int_{X} \prod_{i=1}^{n} x_{i}^{2} \tau\left(M, e^{x_{i}}\right) .
$$

This makes sense: for $b_{1}(M) \geq 2$, the relationship between the Milnor torsion and the Alexander polynomial (11) and the fact that $\Delta\left(M, t_{j}\right)$ is regular as the $t_{j} \rightarrow 1$ guarantees that (24) becomes (22), while for $b_{1}(M)=1$ one makes use of (13) to show that (24) becomes (23).

Let us now consider some $N=4$ gauge theory and the topological twist of this microscopic theory. We can refer to all of the equations that one arrives at on the topological gauge theory side as generalized Seiberg-Witten equations. This includes equations that may not involve any matter fields at all (as for the Casson invariant and its generalizations). Let $S W^{G}$ denote the invariant obtained from the generalized Seiberg-Witten equations (formally, as mentioned before, this is the signed sum $[11,14]$ of Euler characteristics of the solution space).

Now consider the low-energy description of this theory, in terms of a supersymmetric sigma model with target space the hyper-Kähler Coulomb branch $X$. Equating the generalized SW invariant and the RW invariant we thus arrive at the

Conjecture: For a connected, compact, closed oriented 3-manifold, M, with $b_{1}(M) \geq 1$, the generalized Seiberg-Witten invariant is

$$
\mathrm{S} W^{G}(M)=\int_{X} \prod_{i=1}^{n} x_{i}^{2} \tau\left(M, e^{x_{i}}\right) .
$$

Put another way: The generalized Seiberg-Witten invariants are given entirely in terms of Milnor Torsion. But by the Meng-Taubes theorem this 
means that they are determined by the Abelian SW invariant $\underline{\mathrm{SW}}(\mathrm{t})$. Consequently, it appears that neither the generalised SW nor the Casson type invariants shed new light on 3-manifolds with $b_{1} \geq 1$.

\section{2 $b_{1}(M)=0$ and Abelian Higher Rank Seiberg-Witten In- variants}

We have seen above that the only real case of interest for the types of invariants we have been considering is the notoriously subtle case of rational homology spheres, $b_{1}(M)=0$. Since there is only one new perturbative invariant for $n=2$ (and also for $n=3$ ) all theories with an 8 (12) dimensional Coulomb branch yield essentially the same invariant (ignoring lower order invariants). The 3 (4) -monopole $S U(2)$ moduli space is believed to correspond to the $S U(3)(S U(4))$ Casson invariant.

However, Abelian theories are usually easier to get a handle on than non-Abelian ones. It is thus reasonable to try to probe the higher order Rozansky-Witten invariants for rational homology spheres with $N=4 U(1)^{r}$ gauge theories coupled to various charged hypermultiplets. We will simply refer to the gauge theoretic equations that the topological field theory leads to as Abelian Seiberg-Witten equations.

To lead to 'useful' generalizations of the standard SW equations, these theories should satisfy the following three conditions:

1. First of all, the resulting SW equations should not be equivalent to a set of $r$ decoupled ordinary SW equations, as in that case one would only probe the already well known term $\sim \mathbf{e}(X) \lambda(M)^{r}$ in the expansion of the RW invariant.

2. Alternatively and equivalently, the matter content will have to be chosen appropriately to ensure that the Coulomb branch moduli space has a metric with some of the $B_{i}(X)(21)$ other than $\mathbf{e}(X)$ non-zero.

3. Finally, in order to have a well defined counting problem on the SW side, one would like the moduli space to be compact. In practice this can be established most readily if an analogue of the Weitzenböck argument of [4] can be used to bound the norm of the spinors and the gauge field strenghts in terms of the scalar curvature of the 3-manifold. This, together with an argument about reducible solutions, is sufficient to establish compactness of the moduli space. 
One Abelian Seiberg-Witten system that might be of interest is that obtained from the twist of the $N=4$ supersymmetric $U(1)^{r}$ theory with $r$, appropriately charged, massless hypermultiplets studied e.g. in [30, 31]. The Coulomb branch of these theories is a multi-dimensional version of the Taub-NUT metric first obtained in [32] as a particular $S U(r+2)$ monopole moduli space.

The corresponding SW equations are special cases of a more general system of equations for gauge group $U(1)^{r}$ coupled to $r$ charged hypermultiplets. These equations are, with $i, j=1, \ldots, r$,

$$
\begin{aligned}
F_{\mu \nu}^{i} & =-\frac{i}{2} \sum_{j}\left(\bar{M}_{j} \sigma_{\mu \nu} M_{j}\right) E_{j i} \\
\not D\left(\sum_{j} E_{i j} A_{j}\right) M_{i} & =0 \quad(\text { no sum over } i)
\end{aligned}
$$

where the $E_{i j}$ are the $j$-th charges of the $i$-th monopole. Under suitable conditions on the charge matrix $E_{i j}$ it is possible to establish bounds on the norms of the $M_{i}$ and $F$ using the Weitzenböck arguments of [4]. For example, for $r=2$ we find that a sufficient condition is

$$
\operatorname{det} E \neq 0 \text {. }
$$

Moreover, it is still true that there are no non-trivial solutions to the equations if $M$ admits a metric with scalar curvature $R>0$, just as for the $U(1)$ SW equations. To establish that these equations are not equivalent to a pair of uncoupled SW equations, and hence really probe the higher order RW invariants, it is e.g. sufficient to show that the integral of $\operatorname{Tr} R_{X}^{4}, R_{X}$ the Riemann curvature two-form of the Coulomb branch moduli space $X$, is non-zero. We will describe these and other aspects of the problem in [33].

\section{Note Added in Proof}

Since submitting this article L. Nicolaescu has, for rational homology spheres, announced a proof of the equivalence of the Seiberg-Witten invariants and the Casson invariant up to terms involving Reidemeister torsion [34].

\section{Acknowledgements}

We are grateful to M.S. Narasimhan for discussions. This work was supported in part by the EC under the TMR contract ERBFMRX-CT96-0090. 


\section{References}

[1] E. Witten, Topological Yang-Mills Theory, Commun. Math. Phys. 117 (1988) 353.

[2] D. Birmingham, M. Blau, M. Rakowski, G. Thompson, Topological Field Theory, Physics Reports 209 Nos. $4 \& 5$ (1991) 129-340.

[3] N. Seiberg, E. Witten, Monopole Condensation, And Confinement In $N=2$ Supersymmetric Yang-Mills Theory, Nucl.Phys. B426 (1994) 19-52; Erratum: ibid. B430 (1994) 485-486, hep-th/9407087; Monopoles, Duality and Chiral Symmetry Breaking in $N=2$ Supersymmetric QCD, Nucl.Phys. B431 (1994) 484-550, hep-th/9408099.

[4] E. Witten, Monopoles and Four-Manifolds, Math.Res.Lett. 1 (1994) 769-796, hep-th/9411102.

[5] M. Mariño, G. Moore, 3-manifold topology and the Donaldson-Witten partition function, Nucl.Phys. B547 (1999) 569-598, hep-th/9811214.

[6] G. Meng, C. Taubes, $\underline{S W}=$ Milnor Torsion, Math. Res. Lett. 3 (1996) 661.

[7] C. Lescop, Global Surgery Formula for the Casson-Walker Invariant, Annals of Math. Studies 140, Princeton Univ. Press, 1996.

[8] V. Turaev, Reidemeister Torsion in Knot Theory, Russian Math. Surveys, 41 (1986) 119-182.

[9] N. Seiberg, E. Witten, Gauge Dynamics And Compactification To Three Dimensions, in The mathematical beauty of physics, Adv. Ser. Math. Phys. 24 (World Scientific, 1997) 333-366, hep-th/9607163.

[10] L. Rozansky, E. Witten, Hyper-Kähler Geometry and Invariants of ThreeManifolds, Selecta Math. (N.S.) 3 (1997) 401-458, hep-th/9612216.

[11] M. Blau, G. Thompson, $N=2$ Topological Gauge Theory, the Euler Characteristic of Moduli Spaces, and the Casson Invariant, Commun. Math. Phys. 152 (1993) 41-72, hep-th/9112012.

[12] C. Vafa, E. Witten, A Strong Coupling Test of S-Duality, Nucl.Phys. B431 (1994) 3-77, hep-th/9408074.

[13] R. Dijkgraaf, G. Moore, Balanced Topological Field Theories, Commun.Math.Phys. 185 (1997) 411-440, hep-th/9608169.

[14] M. Blau, G. Thompson, Aspects of $N_{T} \geq 2$ Topological Gauge Theories and D-Branes, Nucl. Phys. B492 (1997) 545-590, hep-th/9612143.

[15] N. Habegger, G. Thompson, The Universal Perturbative Quantum 3-manifold Invariant, Rozansky-Witten Invariants, and the Generalized Casson Invariant, math/9911049.

[16] K. Walker, An Extension of Casson's Invariant, Annals of Mathematics Studies, 126, Princeton University Press (1992).

[17] K. Intriligator, Compactified Little String Theories and Compact Moduli Spaces of Vacua, hep-th/9909219. 
[18] C. Taubes, Casson's Invariant and Gauge Theory, J. Diff. Geom. 31 (1990) 547.

[19] E. Witten, Topology Changing Amplitudes in 2+1 Dimensional Gravity, Nucl. Phys. B323 (1990) 281.

[20] M. Blau, G. Thompson, Euclidean SYM Theories by Time Reduction and Special Holonomy Manifolds, Phys. Lett. B415, 242-252 (1997), hep-th/9706225.

[21] A. Carey, J. Mc Carthy, B. Wang, R. Zhang, Seiberg-Witten Monopoles in Three Dimensions, Lett. Math. Phys. 39 (1997) 213-228.

[22] Y. Ohta, Topological Field Theories associated with Three Dimensional Seiberg-Witten monopoles, Int. J. Theor. Phys. 37 (1998) 925-956, hep-th/9611120.

[23] G. Thompson, New Results in Topological Field Theory and Abelian Gauge Theory, in 1995 ICTP Summer School in High-Energy Physics and Cosmology (World Scientific, 1997) 194-255, hep-th/9511038.

[24] Y. Lim, The equivalence of Seiberg-Witten and Casson invariants for homology 3-spheres, Math. Res. Lett. 6 (1999), 631-643.

[25] W. Chen, The Seiberg-Witten theory of homology 3-spheres, dg-ga/9703009.

[26] M. Marcolli, Seiberg-Witten gauge theory, Texts and Readings in Mathematics Vol. 17, Hindustan Book Agency, 1999, available from http://www-math.mit.edu/ matilde/.

[27] L. Nicolaescu, Seiberg-Witten theoretic invariants of Lens spaces, Canad. J. Math. 53 (2001) 780-808, math.DG/9901071.

[28] N. Hitchin, J. Sawon, Curvature and characteristic numbers of hyperkähler manifolds, math/9908114.

[29] J. Labastida, M. Mariño, Non-Abelian Monopoles on Four-Manifolds, Nucl. Phys. B448 (1995) 373-398, hep-th/9504010; M. Mariño, The Geometry of Supersymmetric Gauge Theories in Four Dimensions, hep-th/9701128.

[30] K. Intriligator, N. Seiberg, Mirror Symmetry in Three Dimensional Gauge Theories, Phys.Lett. B387 (1996) 513-519, hep-th/9607207.

[31] A. Hanany, E. Witten, Type IIB Superstrings, BPS Monopoles, and Three-Dimensional Gauge Dynamics, Nucl.Phys. B492 (1997) 152-190, hep-th/9611230.

[32] K. Lee, E. Weinberg, P. Yi, The Moduli Space of Many BPS Monopoles for Arbitrary Gauge Groups, Phys.Rev. D54 (1996) 1633-1643, hep-th/9602167.

[33] M. Blau, G. Thompson, in preparation.

[34] L. Nicolaescu, Seiberg-Witten Invariants of Rational Homology Spheres, math.GT/0103020. 